\section{P082 CIRCULATING MIR-145 AS A MARKER OF THERAPEUTIC RESPONSE IN PATIENTS WITH ANKYLOSING SPONDYLITIS RECEIVING ANTI-TNF THERAPY}

${ }^{1} \mathrm{~K}$ Prajzlerová*, ${ }^{1} \mathrm{~V}$ Hrušková, ${ }^{2} \mathrm{M}$ Komarc, ${ }^{1}$ Š Forejtová, ${ }^{1} \mathrm{~K}$ Pavelka, ${ }^{1} \mathrm{~J}$ Vencovský, ${ }^{1} \mathrm{~L}$ Šenolt, ${ }^{1} \mathrm{M}$ Filková. Institute of Rheumatology and Department of Rheumatology, 1st Faculty of Medicine, Charles University; ${ }^{2}$ Department of Antropometrics and Methology, Faculty of Physical Education and Sport, Charles University, Prague, Czech Republic

\subsection{6/annrheumdis-2018-EWRR2018.99}

Introduction The altered expression of miRNAs is involved in the pathophysiology of inflammatory conditions. Moreover, circulating miRNAs appear promising therapeutic and prognostic biomarkers.

Objectives To investigate the effect of anti-TNF therapy on the levels of circulating miRNAs in patients with ankylosing spondylitis (AS).

Methods Our study included 19 AS patients. Clinical and laboratory parameters of disease activity were assessed at baseline (M0) and after 3 (M3) and 12 months (M12) of therapy. Total RNA from plasma was isolated using miRNeasy Serum/ Plasma Kit (Qiagen). First, a comprehensive analysis of miRNAs was performed using TaqMan Low Density Array (TLDA) in 3 patients. Next, single assays were used for validation of selected miRNAs. dCt method was used for relative quantification. Data were analysed using ANOVA with Bonferroni corrections and Spearman's correlation coefficient.

Results All AS patients had high disease activity at baseline and had a good therapeutic response to anti TNF therapy at M3 and M12 (BASDAI p $<0.001$, ASDAS p <0.001).

Out of all 380 miRNAs analysed by TLDA, 125 miRNAs were detected in all samples, 148 miRNAs were detected at M0, 154 at M3 and 151 at M12. Validation of 17 selected miRNAs confirmed significant downregulation of miR-145 at M3 (TLDA 1.59-change; single assays 1.62-change, $p=0.024$ ), but the downregulation did not reach statistical significance at M12 (TLDA 1.46-change; single assays 1.11-change, $\mathrm{p}>0.05$ ).

The decrease in miR-145 expression from M0 to M3 significantly correlated with disease activity improvement over time from M3 to M12 as per BASDAI ( $\mathrm{r}=0.672, \mathrm{p}=0.002)$ and ASDAS scores $(\mathrm{r}=0.607, \mathrm{p}=0.006)$ and VAS at M12 $(r=0.515, p=0.024)$. Although the overall clinical efficacy of anti-TNF therapy sustained, 6 patients revealed some ASDAS worsening at M12. This may have resulted to correlation of higher miR-145 levels at M3 with disease activity worsening as per increase in ASDAS from M3 to M12 ( $r=0.593$, $\mathrm{p}=0.007)$ and higher ASDAS $(\mathrm{r}=0.533, \mathrm{p}=0.019)$ and VAS $(\mathrm{r}=0.564, \mathrm{p}=0.012)$ at M12.

Conclusions We propose that an early change in miR-145 levels may be a predictor for the future outcome of AS patients as it's early decrease after anti-TNF initiation correlated with further disease activity improvement. These data suggest the potential to use circulating miRNAs as biomarkers of treatment response in AS.

Acknowledgements Grants 17-33127A and SVV 260263. Disclosure of interest None declared

\section{P083 COMORBID TNF-MEDIATED HEART VALVE DISEASE AND CHRONIC POLYARTHRITIS SHARE COMMON MESENCHYMAL AETIOPATHOGENESIS}

${ }^{1} \mathrm{~L}$ Ntari* ${ }^{1} \mathrm{M}$ Sakkou, ${ }^{1,2} \mathrm{P}$ Chouvardas, ${ }^{3} \mathrm{I}$ Mourouzis, ${ }^{1} \mathrm{~A}$ Prados, ${ }^{4} \mathrm{MC}$ Denis, ${ }^{4} \mathrm{~N}$ Karagianni, ${ }^{3} \mathrm{C}$ Pantos, ${ }^{1,2} \mathrm{G}$ Kollias. ${ }^{1}$ Institute of Immunology, Biomedical Sciences Research Centre (BSRC), 'Alexander Fleming', Vari, Athens; ' Department of Physiology; ${ }^{3}$ Department of Pharmacology, Medical School, National Kapodistrian University, Athens; ${ }^{4}$ Biomedcode Hellas SA, Vari, Athens, Greece

\subsection{6/annrheumdis-2018-EWRR2018.100}

Objectives Rheumatoid arthritis (RA) is a chronic condition characterised by prolonged inflammation of the joints leading to bone and cartilage destruction. The key molecular and cellular regulators of RA pathology are the Tumour Necrosis Factor (TNF) and the mesenchymal-origin Synovial Fibroblasts (SFs), respectively. Apart from the joint pathology, RA patients also show higher morbidity rates, mainly due to the development of extraarticular conditions including cardiovascular, gut and skin disease manifestations. The Tg197 arthritis model develops TNF-driven and mesenchymal synovial fibroblasts (SFs)-dependent polyarthritis. Here, we investigate whether this model develops, similarly to human patients, co-morbid heart pathology and we explore cellular and molecular mechanisms linking arthritis to cardiovascular comorbidities.

Methods The established human TNF-transgenic model of arthritis (Tg197) was used to evaluate possible arthritis-related cardiovascular disease. We further studied the role of Valve Interstitial Cells (VICs) in the pathology, using the ColvI-Cre mouse which specifically targets mesenchymal cells. Tg197 ColVI-Cre Tnfr ${ }^{\mathrm{fl} / \mathrm{fl}}$ and Tg197 ColVI-Cre Tnfr $1^{\text {cneo/cneo }}$ mice were used to explore the role of mesenchymal TNF signalling in the developing heart valve disease. Similarities of pathogenic VICs and SFs were analysed by RNA-sequencing.

Results Tg197 mice develop left-sided heart valve disease, characterised by valvular fibrosis with minimal signs of inflammatory cell infiltration and thickened areas, consisting almost entirely of ColVI-expressing mesenchymal VICs, indicating proliferation of this cell type as a hallmark of the observed phenotype. Development of the pathology results in valve stenosis and left ventricular dysfunction, accompanied by arrhythmic episodes and, occasionally, valvular regurgitation. TNF-dependency of the pathology was indicated by the disease amelioration following pharmacological inhibition or genetic ablation of TNF-signalling. Interestingly, Tg197-derived VICs exhibited an activated phenotype ex vivo, resembling the activation of pathogenic Tg197-derived SFs. A significant functional correlation between these two mesenchymal cells was further supported by RNA-seq analysis, suggesting common pathogenic cellular mechanisms operating in arthritis and cardiovascular comorbidities.

Conclusions TNF-mediated joint pathologies, commonly associated with comorbid heart valve disease, are efficiently modelled in Tg197 arthritis model and are commonly underlined by mesenchymal cell-specific pathogenic mechanisms.

Disclosure of interest None declared 